\title{
Laypersons' Priority-Setting Preferences for Allocating a COVID-19 Patient to a Ventilator: Does a Diagnosis of Alzheimer's Disease Matter?
}

This article was published in the following Dove Press journal:

Clinical Interventions in Aging

\author{
Perla Werner (iD) \\ Ruth Landau ${ }^{2}$ \\ 'Department of Community Mental \\ Health, University of Haifa, Haifa, Israel; \\ ${ }^{2}$ School of Social Work, Hebrew \\ University, Jerusalem, Israel
}

\begin{abstract}
Purpose: The current study aimed 1) to assess laypersons' priority-setting preferences for allocating ventilators to COVID-19 patients with and without AD while differentiating between a young and an old person with the disease, and 2) to examine the factors associated with these preferences.
\end{abstract}

Methods: A cross-sectional online survey was conducted among a sample of 309 Israeli Jewish persons aged 40 and above.

Results: Overall, almost three quarters (71\%) of the participants chose the 80 -year-old patient with a diagnosis of AD to be the last to be provided with a ventilator. The preferences of the remaining quarter were divided between the 80 -year-old person who was cognitively intact and the 55-year-old person with AD. Education and subjective knowledge about AD were significantly associated with participants' preferences.

Conclusion: Our results suggest that cognitive status might not be a strong discriminating factor for laypersons' preferences for allocating ventilators during the COVID-19 pandemic.

Keywords: pandemic, dementia, care rationing, prioritizing

\section{Introduction}

COVID-19, the disease caused by the novel coronavirus SARS-CoV-2, originated in Wuhan, China, in December 2019. Since then the virus has spread rapidly across the world, and on March 12 $2^{\text {th }}, 2020$, with more than 20,000 confirmed cases and almost 1000 deaths in Europe alone, the World Health Organization defined COVID-19 as a pandemic. ${ }^{1}$

As demonstrated in previous pandemics, such as the SARS pandemic, ${ }^{2}$ health systems at times of crisis confront many challenges, including the need to allocate limited resources to increasing numbers of people who need them. This task, called priority setting, refers to the process of allocating resources that are scarce while relying on the highest scientific and moral considerations to do so. ${ }^{3}$ The need for a fair allocation of resources that are in short supply has been evident during the ongoing COVID-19 crisis, and includes attaining and distributing enough N-95 masks and other personal protective equipment for healthcare forces, ${ }^{4}$ as well as intensive care unit (ICU) beds for people with the disease. $^{5}$ Specifically, due to the threat of their shortage and their potential lifesaving nature, the decision to allocate or reallocate ventilators is accompanied by serious medical and ethical dilemmas. ${ }^{5-8}$
Correspondence: Perla Werner Department of Community Mental Health, University of Haifa, Mt. Carmel, Haifa, Israel Tel +972-54-3933066

Email pwerner@univ.haifa.ac.il 
In order to attain a fair allocation of scarce resources while responding rapidly and effectively to the crisis, guidelines and protocols have been developed in many countries. A recent review of 27 relevant guidelines published in different states in the US showed that the majority of them do not give priority to any specific group for the allocation of ventilators. However, when tiebreakers or withdrawal decisions have to be made, several factors including age or existing conditions such as cognitive deterioration might be taken into account. ${ }^{9}$ This is a worrisome prospect, as it is clear today that compared to younger individuals, older individuals, and especially those with underlying chronic conditions such as diabetes and hypertension, are at a higher risk of developing COVID-19, as well as being at a much higher risk of mortality. ${ }^{10}$ These risk levels might be even higher for individuals with Alzheimer's disease (AD), for several reasons. First, older people with $\mathrm{AD}$ are at a higher risk of having comorbid diseases than are older people without $\mathrm{AD},{ }^{11}$ making them a clear target for developing serious complications if they become infected with COVID-19. Second, some people with AD might have difficulty understanding and following the most basic recommendations for preventing the spread of the virus, such as handwashing, using a mask, and social distancing. ${ }^{12}$

However, ruling out the right to be considered eligible for scarce resources only on the basis of a diagnosis of $\mathrm{AD}$, is “ . . unjustified discrimination" (p.8), ${ }^{13}$ especially as it is known today that persons with $A D$ vary in the presentation and severity of the disease, in their decision-making capability, and in the possibility to live many years in good quality. ${ }^{14}$ Concerned by potential disadvantages in the allocation of resources, Alzheimer Europe urged health systems to recognize that people with $\mathrm{AD}$ "can live many years ... with a high quality of life" and therefore should not be refused treatment because of their diagnosis. ${ }^{15}$ Similarly, Alzheimer's Disease International claimed that, "Decisions should not be made based purely on age or the existence of a condition like dementia, which should be in violation of that individual's human rights"16

Although ultimately pronouncements regarding the allocation of resources are in the hands of clinicians, there is a growing consensus in the literature that engaging the public in the process, or at least understanding its preferences, is important for attaining the best decisions and supporting them across time. ${ }^{3}$ In line with this idea, the first aim of this study was to assess laypersons' preferences for allocating ventilators to an older and a younger person with $\mathrm{AD}$, as well as to an older person without $\mathrm{AD}$. Based on the maximizing life-years and maximizing social benefit principles ${ }^{4,17}$ suggesting that adopting age as a criterion for rationing purposes is a moral decision, together with the noticeable discourse on professional platforms as well as in the media about increased ageism during the COVID-19 pandemic, ${ }^{18-22}$ we hypothesized that laypersons would prefer to allocate a ventilator first to the 55-year-old person with an $\mathrm{AD}$ diagnosis and last to the 80-year-old person with an $\mathrm{AD}$ diagnosis. Our second aim was to examine the factors associated with these preferences. Based on a previous study about laypersons' views on priority setting for people with $\mathrm{AD},{ }^{23}$ we expected to find a significant association with sociodemographic characteristics, moral justifications, and beliefs about AD. Beliefs about COVID-19 were also examined due to their relevance to the topic, but no hypothesis was stated regarding these beliefs.

\section{Methods}

\section{Design and Sample}

A cross-sectional survey was conducted among a sample of 309 Israeli Jewish individuals aged 40 and above. This age group was selected for two reasons: a) there is a clear association between increased age and becoming seriously ill or dying from COVID-19, and b) the incidence rates of cognitive impairment increase with age, mainly after age 40, making the topic of the study especially relevant to this age group. Potential participants were recruited from a comprehensive internet survey panel through a major Israeli internet panel company (PanelView) with extensive experience in academic surveys. Potential respondents were invited to participate in an online survey for a limited period (June 8 till June 9, 2020). Once quotas by gender and age were reached for each parameter, the survey was closed. Respondents received small redeemable rewards in exchange for their participation.

\section{Measures}

We used a structured questionnaire including the following measures:

\section{Dependent Variable}

\section{Preferences for Allocating Ventilators}

Participants were presented with the description of three fictional male COVID-19 patients, varying in their age and cognitive status. Moses was a 55-year-old man with 
a diagnosis of $\mathrm{AD}$, Jacob was an 80-year-old man with a diagnosis of $\mathrm{AD}$, and Samuel was an 80-year-old cognitively intact man. Our rationale for comparing a young and old person with $\mathrm{AD}$ was that a previous study had shown that laypersons' attitudes differed based on the age of the person with $\mathrm{AD} .^{24}$ All patients were presented as being married and having three children, living at their homes with their spouses, and having a diagnosis of diabetes and high blood pressure. Participants were randomly presented with an item asking them which of the three fictional patients should in their opinion be allocated a ventilator first/last. Once a response was given, participants were asked which of the remaining two patients should be allocated to a ventilator first/last.

\section{Independent Variables}

As stated above, the selection of these variables was based on a previous study conducted by our group. ${ }^{23}$

\section{Sociodemographic Characteristics}

These included gender, age, education (less than 16 years of education, $16+$ years of education), marital status (single or widowed vs married), and religiosity (secular vs traditional or religious or Orthodox).

\section{Moral Reasoning}

Participants were asked to report the importance they attributed to 10 items reflecting principles and rationales for priority setting. ${ }^{25}$ Each item was rated on a Likert-type scale ranging from 1 (not at all important) to 5 (very important). An example item is "Everyone deserves the same chance of rescue from-threatening circumstances". An overall index was calculated by averaging the items. The index showed good internal reliability (Cronbach's alpha $=0.73$ ).

\section{AD Variables}

As in previous research, ${ }^{26}$ these variables included susceptibility to and fear of getting $\mathrm{AD}$, subjective knowledge about $\mathrm{AD}$, familiarity with $\mathrm{AD}$, and stigmatizing beliefs towards a person with AD.

Susceptibility to Developing AD. Participants were asked to report their perceived risk of developing $\mathrm{AD}$ during their lifetime, using one item rated on a 5-point Likerttype scale, ranging from 1 (no risk at all) to 5 (very high risk).

Fear About Developing AD. Participants were asked to report their perceived fear about developing AD during their lifetime, using one item rated on a 5-point Likert-type scale, ranging from 1 (no fear at all) to 5 (very great fear). Subjective Knowledge About AD. Was assessed with a single question: "How much do you know about AD?" Answers were rated on a 5-point Likert-type scale, ranging from 1 (not much at all) to 5 (very much).

Familiarity with $A D$. Was assessed by asking the participants whether they know someone with the disease.

Stigmatic Beliefs Towards a Person with AD. We examined participants' negative and positive emotional reactions, as well as their discriminatory behavior towards a person with $\mathrm{AD}$.

Emotional Reactions Towards a Person with AD. Participants were asked to report their emotional reactions towards a person with $\mathrm{AD}$ using a list of seven emotions: fear, pity, uneasiness, desire to help, concern, disgust, and ridicule. Each item was rated on a five-point Likert-type scale ranging from 1 (not at all) to 5 (extremely). Two indices (for negative and positive emotions) were created by averaging the items. These indices showed good to very good internal reliability (Cronbach's alpha $=0.80$ and 0.72 , respectively, for the negative and positive index).

Discriminatory Behavior. Was assessed using 7 items rated on a five-point Likert-type scale ranging from 1 (not at all) to 5 (extremely). An example item was: "People will avoid a person with AD." A composite index was calculated by averaging the items. The index showed excellent internal reliability (Cronbach's alpha = $0.90)$.

COVID-19 Variables. These included susceptibility to and fear of getting COVID-19.

Susceptibility to Developing COVID-19. Participants were asked to report their perceived risk of developing COVID19 using one item rated on a 5-point Likert-type scale, ranging from 1 (no risk at all) to 5 (very high risk).

Fear About Developing COVID-19. Participants were asked to report their perceived fear about developing COVID-19 using one item rated on a 5-point Likert-type scale, ranging from 1 (no fear at all) to 5 (very great fear).

\section{Statistical Analysis}

The data were cleaned, coded, and analyzed using SPSS version 27.0. Descriptive statistics (percentages, means, and standard deviations) were used to describe the sample and the main variables. A multinomial logistic regression was calculated for the preference of receiving the ventilator first, between the three nominal options. Due to the multitude of independent variables they were entered in 
a forward stepwise manner, using most independent variables as continuous, and marital status, religiosity, subjective knowledge of $\mathrm{AD}$, and familiarity as dichotomous. Odds ratios and confidence intervals were calculated. Significance level was set at $\mathrm{p}<0.05$.

\section{Ethical Considerations}

The study's protocol was approved by the Ethics Committee of the University of Haifa (Number 254/20).

As customary with online surveys, ${ }^{27}$ and in compliance with the Declaration of Helsinki, consent was implied upon completion of the survey. To secure potential participants' understanding of their participation, information about the aim and importance of the study was provided in the first page of the survey, together with the option of stopping participation at any time. Moreover, participants were presented with information about how privacy and confidentiality will be protected. These included by securing them that although they will be given an identification code by the company's administrator, this information will not be given to the researchers or to anyone else.

\section{Results}

\section{Participants}

As can be observed in Table 1, although the sample was similarly divided between male and female, as well as among secular and religious participants, the majority were married. The average age was 56, and the participants reported having an average of 15 years of education.

Table I Participants' Characteristics $(n=309)$

\begin{tabular}{|l|l|}
\hline Characteristics & Percentage/Mean \\
\hline Gender (\%) & \\
Female & 52.1 \\
Male & 47.9 \\
\hline Marital status (\%) & \\
Not married & 27.39 \\
Married & 72.60 \\
\hline Religiosity (\%) & \\
Secular & 51.1 \\
Religious (traditional, religious, Orthodox) & 48.9 \\
\hline Mean (SD, range) age & $56.13(10.23,40-84)$ \\
\hline Mean (SD, range) education & $15.13(2.72,8-25)$ \\
\hline
\end{tabular}

\section{Preferences for Allocating a Ventilator}

As seen in Figure 1, participants' preferences for which COVID-19 patient should be allocated a ventilator last, were very clear. As hypothesized, the majority - close to $71 \%$ - of the participants chose the 80-year-old person with $\mathrm{AD}$ to be the last to receive this treatment. Preferences for the person to be allocated a ventilator first were equally divided between the 55-year-old patient with $\mathrm{AD}$ and the 80 -year-old patient who was cognitively intact.

\section{Correlates of Priority-Setting Preferences for Allocating a Ventilator}

Contrary to our assumptions, although the multinomial logistic regression model was found significant $\left(\chi_{(4)}^{2}=17.44, \mathrm{p}=\right.$ $0.002)$, only education $\left(\chi_{(2)}^{2}=11.05, \mathrm{p}=0.004\right)$, and subjective knowledge of $\mathrm{AD}\left(\chi_{(2)}^{2}=6.64, \mathrm{p}=0.036\right)$, were significant determinants of participants' preferences (Table 2). These results mean that those preferring Samuel (aged 80, no $\mathrm{AD}$ ) over the two other hypothetical cases had significantly more years of education $(\mathrm{M}=15.69 \mathrm{SD}=2.86$, vs $\mathrm{M}=$ 14.77 $\mathrm{SD}=2.56$ for Moses, and $\mathrm{M}=14.24 \mathrm{SD}=2.33$ for Jacob). Further, a higher percentage of those preferring Samuel (aged 80, no AD) over Jacob (aged 80, old with $\mathrm{AD})$ reported high subjective knowledge of $\mathrm{AD}$ (56.3\% and $35.1 \%$, respectively).

\section{Discussion}

It is likely that the COVID-19 pandemic will have unprecedented consequences for the diagnosis, treatment, and care of individuals with AD. ${ }^{12}$ One of these consequences might be the unfair allocation of scarce resources such as ventilators to this population. Although ultimately healthcare professionals are the ones who will decide about the allocation of medical resources, an understanding of laypersons' preferences might increase the transparency and accountability of the process while ensuring public trust in the fairness of the decisions. Thus, this study's main question was: What are laypersons' preferences and correlates for the allocation of ventilators to COVID-19 patients with and without a diagnosis of $\mathrm{AD}$ ?

Our results suggest that laypersons widely agree regarding which COVID-19 patient should be allocated a ventilator last. Almost three quarters of the participants chose the 80-year-old patient with a diagnosis of $\mathrm{AD}$ to be the last to be provided with this potentially life-saving treatment. The preferences of the remaining quarter were 
80

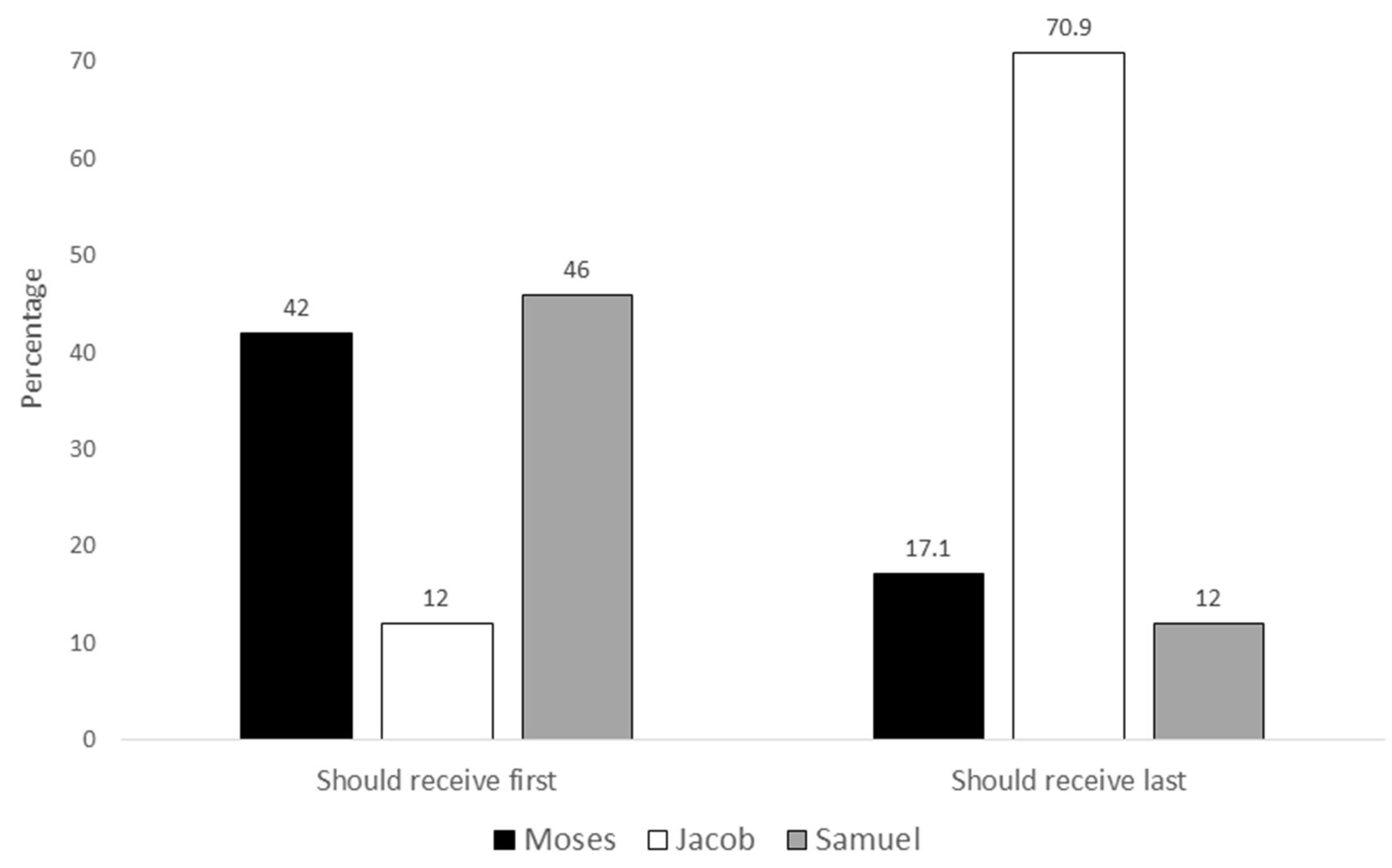

Figure I Preferences for allocating a ventilator (\%).

divided between the 80 -year-old person who was cognitively intact and the 55-year-old person with cognitive deterioration, hinting that laypersons' preferences are not based solely on an age criterion, but on the combination of assessing the patient's life expectancy as a result of age and as a result of an underlying disease, such as AD. This result is compatible with the published literature as well as with policy-makers' recommendations. Recently, Kim \& Grady ${ }^{13}$ strengthened the need to adhere during the COVID-19 crisis to the main ethical principles guiding the allocation of scarce resources when considering the needs of people with cognitive deterioration. More specifically, they stated the need for clinicians to base their decisions on the values of fairness and respect regardless of the type of impairment or of their own evaluation about the patient's quality of life. Similarly, an Israeli committee's position paper on the subject of priority setting of seriously ill COVID-19 patients, stipulates that there should be no discrimination on anything other than decisions made on a medical basis. ${ }^{28}$ Our findings suggest that the Israeli public might be prepared to accept these requirements with legitimacy and confidence.
None of the examined factors - including sociodemographic characteristics, moral justifications, or beliefs about AD and COVID-19 - was associated with participants' choices. A similar finding was reported by ${ }^{17}$ reinforcing the argument that during a crisis laypersons rely almost totally on assessments of the patient's survivability chances. This is an encouraging finding as the ability to strengthens survivability has been found to be one of the cornerstones of ethical prioritization. ${ }^{29}$ Although this preference might not create conflicts among laypersons and the healthcare professionals who are making the decisions, it still requires that professionals be trained in delivering difficult and distressing news with empathy and care even in times of emergency such as the COVID-19 pandemic.

When asked to make decisions regarding which patient should be allocated a ventilator first, participants' preferences were almost equally divided between the 80-yearold person who was cognitively intact and the 55-year-old person with $\mathrm{AD}$. This finding reflects the complexity of making priority-setting life-and-death decisions. Recognizing this difficulty, even for healthcare professionals, scoring systems such as the Sequential Organ 
Table 2 Multinomial Stepwise Logistic Regression of Preferences for Allocating a Ventilator First $(n=309)$

\begin{tabular}{|c|c|c|c|c|}
\hline & B (SE) & OR & $\mathbf{p}$ & $95 \% \mathrm{Cl}$ \\
\hline & \multicolumn{4}{|c|}{ Moses (young with AD) vs Samuel (old no AD) } \\
\hline Education years & $-0.12(0.05)$ & 0.89 & 0.013 & $0.8 \mathrm{I}, 0.97$ \\
\hline \multirow[t]{2}{*}{ Subjective knowledge of $A D$} & $-0.38(0.25)$ & 0.68 & 0.123 & $0.42,1.1 \mathrm{I}$ \\
\hline & \multicolumn{4}{|c|}{ Jacob (old with $A D$ ) vs Samuel (old no AD) } \\
\hline Education years & $-0.21(0.08)$ & 0.81 & 0.008 & $0.69,0.94$ \\
\hline \multirow[t]{2}{*}{ Subjective knowledge of $A D$} & $-0.96(0.40)$ & 0.38 & 0.017 & $0.17,0.84$ \\
\hline & \multicolumn{4}{|c|}{ Jacob (old with $A D$ ) vs Moses (young with $A D$ ) } \\
\hline Education years & $-0.09(0.08)$ & 0.91 & 0.232 & $0.78,1.06$ \\
\hline Subjective knowledge of $A D$ & $-0.58(0.40)$ & 0.56 & 0.149 & $0.26,1.23$ \\
\hline
\end{tabular}

Failure Assessment (SOFA) were developed. ${ }^{9}$ Based on an assessment of major functioning systems (such as heart, lungs, kidneys, liver, and blood), patients with higher SOFA scores would be given a lower priority to be allocated a ventilator. However, the accuracy of such scoring systems in predicting survivability is being debated, and it has been suggested that other factors might need to be considered when making the decision of to whom a ventilator should be allocated, and more importantly, of who to remove from such treatment. ${ }^{30}$ Our findings suggest that laypersons might share similar or even more difficult dilemmas. For example, it seems that the participants in our study were unable to discriminate between the chances for survivability of an older person who was cognitively intact and a young person with cognitive deterioration. Alternatively, our findings suggest that the participants did not base their preferences on chronological age as a sole and defining discriminating factor. The diagnosis of $\mathrm{AD}$, as expected by leading associations in the area, ${ }^{15}$ was also not used as the sole, deciding factor. These findings are similar to those reported by two studies assessing the general public's preference for allocating scarce resources during times of disaster. ${ }^{31,32}$ According to these researchers, in times of crisis and emergency, such as a pandemic, laypersons' preferences are guided only by the principle of saving the largest number of lives possible. This finding might also explain the few significant associations we found between the dependent variables - that is, preferences for the allocation of ventilators - and the wide variety of independent variables we examined. Indeed, higher education and higher levels of subjective AD knowledge were the only significant factors related to increased preference for the 80 -year-old patient without cognitive impairment to be allocated a ventilator first. These findings might stem from lower levels of ageism found in these groups. ${ }^{33,34}$ Alternatively it might be associated to higher levels of subjective knowledge about $\mathrm{AD}$ being associated to more positive attitudes of persons expressing their healthcare preferences early on, either through informal conversations with family members and practitioners or through the completion of advance care planning. ${ }^{35}$ However, we are unable to empirically confirm this assumption as, regrettably, ageist beliefs were not assessed in this study.

\section{Limitations}

Several limitations should be taken into account. First, the study's cross-sectional design does not allow us to draw causal conclusions. Second, our sample included only Jewish participants, therefore limiting the study's generalizability. Third, respondents were recruited from an adult panel for web surveys taken from a marketing agency. As such, we are unable to discount the effects of selfselection. Fourth, although we used structured validated measures, we nevertheless relied on self-reported data, which may have resulted in an increased social desirability bias. Fifth, we did not include a 55-year-old patient who had COVID-19 and was cognitively intact, therefore limiting our ability to better assess the impact of the age criterion. Finally, our study examined priority-setting preferences only for allocating a ventilator. Making the decision to remove a patient from a ventilator might pose even greater dilemmas and stress. ${ }^{7}$ Indeed, according to the aforementioned Israeli position paper, the matter of taking 
a person off a ventilator is a very controversial issue, and the committee participants' views were equally divided.

\section{Implications and Conclusions}

Despite these limitations, we believe that this study contributes greatly, theoretically and practically, to one of the more difficult decisions confronting healthcare systems worldwide during the COVID-19 crisis - allocating ventilators in the event of their scarcity. Theoretically, it adds to the limited number of empirical studies assessing laypersons' views about priority setting of scarce resources. ${ }^{25,36}$ Expanding the understanding of laypersons' preferences is especially important during the current crisis for several reasons. First, it allows to attain a more transparent and accountable process. Second, it might help increasing the relatively low levels of trust the public has in their government's leaders and decisions, ${ }^{37}$ which in time may improve the public's adherence to preventive measures, such as social distancing and using a mask, and decrease the numbers of those affected by the virus. Finally, becoming familiar with the public's preferences for allocating scarce resources might guide policy-makers and clinicians to improve their decision-making and communication processes.

Practically, by suggesting that age and cognitive status might not be in the eyes of laypersons such strong discriminating factors as assumed, our results might ease policy-makers regarding the acceptance and legitimacy of their decisions. We suggest policy-makers and health care organizations to advocate clearly and openly for nondiscriminatory triage protocols based on age or a diagnosis of dementia. This will require clinicians to examine carefully the functioning and morbidity status of each patient, as well as their wishes and preferences, including the availability of advance care directives. Given that such detailed and intense process might be difficult for clinicians at emergency times, we suggest that triage decisions should be made by a team or a committee in order to ease the burden of bed-side clinicians. ${ }^{14}$ Finally, similar to others, ${ }^{17}$ our study showed that associations between laypersons' preferences and their sociodemographic characteristics, moral justifications, and beliefs were modest and few. Future studies, using a qualitative methodology, should further explore what are laypersons' motivations for their allocation preferences.

\section{Data Sharing Statement}

The data that support the finding of this study are available from the corresponding author upon reasonable request.

\section{Funding}

This research received no specific grant from any funding agency in the public, commercial, or not-for-profit sectors.

\section{Disclosure}

The authors declare that there is no conflict of interest.

\section{References}

1. World Health Organization. WHO Announces COVID-19 Outbreak a Pandemic. 2020. Available from: www.WHO.int/emergencies/dis eases/novel-coronavirus-2019. Accessed December 14, 2020.

2. Bell JAH, Hyland S, DePellegrin Upshur T, et al. SARS and hospital priority setting: a qualitative case study and evaluation. BMC Health Serv Res. 2004;4(1):36. doi:10.1186/1472-6963-4-36

3. Chalkidou K, Glassman A, Marten R, et al. Priority-setting for achieving universal health coverage. Bull World Health Organ. 2016;94(6):462-467. doi:10.2471/BLT.15.155721

4. Emanuel EJ, Persad G, Upshur R, et al. Fair allocation of scarce medical resources in the time of Covid-19. N Engl J Med. 2020;382 (21):2049-2055. doi:10.1056/NEJMsb2005114

5. Peterson A, Emanuel EAL, Hart R, et al. Ethics of reallocating ventilators in the covid-19 pandemic. BMJ. 2020;369:m1828. doi:10.1136/bmj.m1828

6. Pathak PA, Sönmez T, Ünver U, et al. Leaving No Ethical Value Behind: Triage Protocol Design for Pandemic Rationing. The National Bureau of Economic Research;2020. doi:10.3386/w26951

7. Truog RD, Mitchell C, Daley GQ. The toughest triage - allocating ventilators in a pandemic. $N$ Engl J Med. 2020;382(21):1973-1975. doi:10.1056/nejmp2005689

8. White DB, Lo BA. A framework for rationing ventilators and critical care beds during the COVID-19 pandemic. JAMA. 2020;323 (18):1773-1774. doi:10.1001/jama.2020.5046

9. Piscitello GM, Kapania EM, Miller WD, et al. Variation in ventilator allocation guidelines by US State during the coronavirus disease 2019 pandemic: a systematic review. JAMA. 2020;3(6):e2012606. doi:10.1001/jamanetworkopen.2020.12606

10. Landry MD, van den Bergh G, Hjelle KM, et al. Betrayal of trust? The impact of the COVID-19 global pandemic on older persons. $J$ Appl Gerontol. 2020;39(7):687-689. doi:10.1177/0733464820924131

11. Clague F, Mercer SW, McLean G, et al. Comorbidity and polypharmacy in people with dementia: insights from a large, population-based cross-sectional analysis of primary care data. Age Ageing. 2017;46(1):33-39. doi:10.1093/ageing/afw176

12. Brown EE, Kumar S, Rajji TK, et al. Anticipating and mitigating the impact of the COVID-19 pandemic on alzheimer's disease and related dementias. Am J Geriatr Psychiatry. 2020;28(7):712-721. doi:10.1016/j.jagp.2020.04.010

13. Kim SYH, Grady C. Ethics in the time of COVID: what remains the same and what is different. Neurology. 2020;94:1007-1008. doi:10.1212/WNL00000000009520

14. Smith EE, Couillard P, Fisk JD, et al. Pandemic dementia scarce resource allocation. Can Geriatr J. 2020;23(3):216-218. doi:10.5770/cgj.23.457

15. Alzheimer Europe. Position Regarding the Allocation of Scarce Resources for Intensive Care Services During the COVID-19 Pandemic, Luxembourg: Alzheimer Europe. 2020. Available from: https://www.alz heimer-europe.org/Policy/Our-opinion-on/Triage-decisions-duringCOVID-19-pandemic. Accessed December 14, 2020. 
16. Barbarino P, Lynch C, Bliss A, et al. From Plan to Impact III: Maintaining Dementia as a Priority in Unprecedented Times. London: Alzheimer's Disease International; 2020.

17. Huseynov S, Palma MA, Nayga RM Jr. General public preferences for allocating scarce medical resources during COVID-19. Available from: https://www.resaerchgate.net/profile/Samir_Huseynov3/publica tions/341992148_General_Public_Preferences_Allocating_Scarce_ Medical_Resources. Accessed November 22, 2020.

18. Ayalon L, Chasteen A, Diehl M, et al. Aging in times of the COVID-19 pandemic: avoiding ageism and fostering intergenerational solidarity. J Gerontol B Psychol Sci Soc Sci. 2020:1-4. doi:10.1093/geronb/gbaa051

19. Brooks J, Jackson D. Older people and COVID-19: isolation, risk and ageism. J Clin Nurs. 2020;29(13-14):2044-2046. doi:10.1111/ jocn. 15274

20. Cesari M, Proietti M. COVID-19 in Italy: ageism and decision making in a pandemic. $J$ Am Med Dir Assoc. 2020;21(5):576-577. doi:10.1016/j.jamda.2020.03.025

21. Cipriani G, Di Fiorino M. Access to care for dementia patients suffering from COVID-19. Am J Geriatr Psychiatry. 2020;28 (7):796-797. doi:10.1016/j.jagp.2020.04.009

22. Fraser S, Lagacé M, Bongué B, et al. Ageism and COVID-19: what does our society's response say about us? Age Ageing. 2020;49 (5):692-695. doi:10.1093/ageing/afaa097

23. Werner P. Israeli lay persons' views on priority-setting criteria for alzheimer's disease. Health Expect. 2009;12(2):187-196. doi:10.1111/j.1369-7625.2008.00523.x

24. Werner P, Raviv-Turgeman L, Corrigan PW. The influence of the age of dementia onset on college students' stigmatic attributions towards a person with dementia. BMC Geriatr. 2019;20:104. doi:10.1186/ s12877-020-1505-4

25. Denburg AE, Ungar WJ, Chen S, et al. Does moral reasoning influence public values for health care priority setting?: a population-based randomized stated preference survey. Health Policy (New York). 2020;124(6):647-658. doi:10.1016/j. healthpol.2020.04.007

26. Werner P. Discriminatory behavior towards a person with alzheimer's disease: examining the effects of being in a nursing home. Aging Ment Health. 2008;12(6):786-794. doi:10.1080/13607860802380649
27. Eynon R, Fry J, Schroeder R. The ethics of online research. In: Fielding N, Lee R, Blank G, editors. The Sage Handbook of Online Surveys. 2nd. 2016;19-37.

28. Ministry of Health. Position Paper: The Subject of Priority Setting of Seriously Ill COVID - 19 Patients During the COVID - 19 Pandemic. A joint committee of the National Bioethics Committee, the Ethics Committee of the Israeli Medical Association and the Ministry of Health;2020.

29. Ghanbari V, Ardalan A, Zareiyan A, et al. Ethical prioritization of patients during disaster triage: a systematic review of current evidence. Int Emerg Nurs. 2019;43:126-132. doi:10.1016/j. ienj.2018.10.004

30. Pathak PA, Sönmez T, Ünver U. Improving ventilator rationing through collaboration with experts on resource allocation. JAMA. 2020;3(6):e2012838. doi:10.1001/jamanetworkopen.2020.12838

31. Biddison ELD, Gwon HS, Schoch-Spana M, et al. Scarce resource allocation during disasters: a mixed-method community engagement study. Chest. 2018;153(1):187-195. doi:10.1016/j.chest.2017.08.001

32. Li-Vollmer M, Beebe A, Kite H, et al. Public Engagement Project on Medical Service Prioritization During an Influenza Pandemic. Public Health-Seattle \& King County;2009.

33. Chang CY, Hsu HC. Relationship between knowledge and types of attitudes towards people living with dementia. Int J Environ Res Public Health. 2020;17(11):3777. doi:10.3390/ijerph17113777

34. Officer A, Thiyagarajan JA, Schneiders ML, et al. Ageism, healthy life expectancy and population ageing: how are they related? Int J Environ Res Public Health. 2020;17(9):3159. doi:10.3390/ ijerph17093159

35. Manafò E, Peterman L, Vandall-Walker V, et al. Patient and public engagement in priority setting: a systematic rapid review of the literature. PLoS One. 2018;13(3):e0193579. doi:10.1371/journal. pone.0193579

36. Werner P, Kermel Schiffman I. Attitudes and completion of advance care planning: assessing the contribution of health beliefs about alzheimer's disease among israeli laypersons. Palliat Support Care. 2019;17(6):655-661. doi:10.1017/S1478951519000336

37. Cairney P, Wellstead A. COVID-19: effective policymaking depends on trust in experts, politicians, and the public. Policy Design Pract. 2020;1-14. doi:10.1080/25741292.2020.1837466
Clinical Interventions in Aging

\section{Publish your work in this journal}

Clinical Interventions in Aging is an international, peer-reviewed journal focusing on evidence-based reports on the value or lack thereof of treatments intended to prevent or delay the onset of maladaptive correlates of aging in human beings. This journal is indexed on PubMed Central, MedLine, CAS, Scopus and the Elsevier
Bibliographic databases. The manuscript management system is completely online and includes a very quick and fair peer-review system, which is all easy to use. Visit http://www.dovepress.com/ testimonials.php to read real quotes from published authors. 